\title{
THERAPEUTIC POTENTIAL OF „DERIVED- MULTIPLE ALLOGENEIC PROTEINS PARACRINE SIGNALING-D-MAPPS” IN THE TREATMENT OF DRY EYE DISEASE
}

\author{
Carl Randall Harrell ${ }^{1}$, Crissy Fellabaum ${ }^{1}$, Dragica Miloradovic ${ }^{2}$, Aleksandar Acovic ${ }^{3}$, Dragana Miloradovic ${ }^{4}$, Bojana Simovic Markovic ${ }^{2}$, \\ Nebojsa Arsenijevic ${ }^{2}$, Paul Karpecki ${ }^{5}$ and Vladislav Volarevic ${ }^{2 *}$ \\ ${ }^{1}$ Regenerative Processing Plant, LLC, Palm Harbor, Florida, United States of America \\ ${ }^{2}$ University of Kragujevac, Faculty of Medical Sciences, Department of Microbiology and Immunology, \\ Center for Molecular Medicine and Stem Cell Research, Kragujevac, Serbia \\ ${ }^{3}$ University of Kragujevac, Faculty of Medical Sciences, Department of Dentistry, Kragujevac, Serbia \\ ${ }^{4}$ University of Kragujevac, Faculty of Medical Sciences, Department of Genetics, Kragujevac, Serbia \\ ${ }^{5}$ Optometrist in Private Practice, 120 N Eagle Creek, Lexington, Kentucky, United States of America
}

\section{Corresponding author:}

Prof. dr. Vladislav Volarevic,

Faculty of Medical Sciences, Department of Microbiology and Immunology, Center for Molecular Medicine and Stem Cell Research 69 Svetozar Markovic Street, 34000 Kragujevac, Serbia

Phone: +38134306800

E-mail: drvolarevic@yahoo.com

\section{sciendo}

\author{
UDK: 617.7-002:615.37 \\ 606:615.37
}

Ser J Exp Clin Res 2022; 23(2):167-173

DOI: $10.2478 /$ sjecr-2019-0072

\begin{abstract}
The primary aim of this retrospective study was to estimate significance of determining $C$-reactive protein and procalcitonin for a diagnosis of sepsis in adult patients in early triage. Also, the aim of this study was to measure the sensitivity of the SIRS criteria, PCT and CRP levels and sepsis definitions to identify the most serious sepsis cases in the prehospital setting and at the Emergency Department (ED) triage. All patients were divided into two groups according to specific criteria for defining sepsis. First group (SIRS+ group) of patients were patients with clinically and/or laboratory confirmed sepsis (or systemic inflammatory response syndrome (SIRS) to bacterial infection with different localization). For confirmation of the SIRS we consider positive two or more clinical criteria ( $\geq 2$ clinical criteria). The SIRS criteria use the clinical criteria of the Surviving Sepsis Campaign (SSC) for the SIRS, comprising at least two of the following criteria: $H R>$ $90 / \mathrm{min}, R R>20 / \mathrm{min}$ and temperature $<36^{\circ}$ or $\geq 38.3^{\circ} \mathrm{C}$ and the next laboratory parameters such as leucocytosis $>15 \times 109 / \mathrm{L}$, leucopenia $<4 \times 109 / \mathrm{L},>10 \%$ immature leucocytes. Second group of patients were patients with the SIRS negative criteria as a diagnostic tool (SIRS- group). We have founded that the CRP showed high sensitivity but no specificity in patients with sepsis, but on the other side, the PCT as a diagnostic marker showed a high sensitivity and high specificity in these patients. Also, the PCT is in positive correlation with the SIRS criteria, which could be of a clinical significance in early diagnosis of septic infections.
\end{abstract}

Keywords: sepsis, C-reactive protein, procalcitonin, early diagnostic markers. 


\section{INTRODUCTION}

Dry eye disease (DED) is a common, multifactorial disease of the lacrimal system and ocular surface [1]. Loss of homeostasis, instability and hyperosmolarity of the tears, chronic inflammation in the eye and consequent neurosensory dysfunction are usually manifested by visual disturbance, dryness, grittiness, scratchiness, soreness, irritation, burning, watering, and eye fatigue. Significantly reduced functional visual acuity impairs performance of vision-dependent daily activities (reading, writing, driving), greatly diminishing quality of life of DED patients [1]. Currently, there is no cure for dry eye, and the treatments are directed towards improving the symptoms in order to break the vicious circle of chronic inflammation $[1,2]$. Since clinical manifestations of DED are observed in patients who suffer from chronic inflammatory and systemic autoimmune diseases (Sjögren's Syndrome, Rheumatoid arthritis, Systemic lupus erythematosus), it was postulated that detrimental immune response had a crucially important role in the development and progression of DED [2-6]. Therefore, molecular mechanisms responsible for the induction of inflammatory cascade in the eyes of DED patients have been evaluated by large number of research groups. Considering the important role of inflammation in DED development, the main treatment strategy has shifted from hydration and lubrication of dry ocular surface to the immunomodulation and immunoregulation-approach that should address the main pathologic processes responsible for disease progression [2-5]. However, it should be noted that long-term, systemic use of immunosuppressive drugs may result in the development of severe, secondary immunodeficiency, significantly increasing the risk for the development of infectious diseases and malignancy [6]. Therefore, new remedies for DED treatment should suppress detrimental immune response in the eye without affecting systemic inflammatory response [7].

Due to their capacity for production of immunosuppressive factors, mesenchymal stem cells (MSCs) and their secretome have been considered as potentially new agents in DED therapy since they may, after local application in the eye, regulate detrimental immune response without causing lifetreating systemic immunosuppression [8]. Most recently, it was revealed that MSC-derived exosomes (MSC-Exos) were mainly responsible for beneficial effects of MSC-sourced secretome in alleviation of inflammatory eye diseases [9]. MSC-Exos are nano-sized (30-100nm) vesicles that carry nucleic acids, lipids and proteins (cytokines, chemokines) and are capable to modulate migratory and effector functions of immune cells (T lymphocytes, dendritic cells (DCs), macrophages) which have crucially important pathogenic role in DED development and progression. Since membranes of MSC-Exos are enriched in cholesterol, sphingomyelin, ceramide and lipid raft proteins, these nano-sized carriers of MSC-derived immunosuppressive factors may be taken by target cells through endocytosis or membrane fusion, regardless of biological barriers [9].
In line with these findings, we recently developed immunomodulatory ophthalmic solution "derived- Multiple Allogeneic Proteins Paracrine Signaling (d-MAPPS)" which activity is relied on immunosuppressive capacity of MSCderived secretome [10]. d-MAPPS contains MSC-Exos, growth factors and immunosuppressive cytokines that are able to efficiently suppress generation of inflammatory phenotype in T cells and macrophages [10]. Herewith, we demonstrated that d-MAPPS protected human corneal epithelial cells from chemical injury and efficiently alleviated ocular discomfort and pain in DED patients.

\section{MATERIAL AND METHODS}

\section{Preparation of d-MAPPS samples and eye drops}

Sterile d-MAPPS is a bio-engineered biologic product obtained from amniotic fluid derived MSCs (AF-MSCs), previously collected from healthy human donors. Blood samples were given by the donor prior to or at the time of collection and were tested by laboratories certified under the Clinical Laboratory Improvement Amendments (CLIA) and were found negative using United States (U.S) Food and Drug Administration (FDA) licensed tests for detection of at minimum: Hepatitis B Virus, Hepatitis C Virus, Human Immunodeficiency Virus Types $1 / 2$, Treponema Pallidum. AF samples were obtained with patient consent and kept at $4^{\circ} \mathrm{C}$ until processed. d-MAPPS samples were bio-engineered as AFMSC-derived sterile product containing AF-MSC-Exos and AF-MSC-derived cytokines and growth factors, manufactured under current Good Manufacturing Practices (cGMP), regulated and reviewed by the FDA (10). Sterile d-MAPPS incorporate Regenerative Processing Plant's (RPP) proprietary patented sterilization process to provide for a safe, sterile product. d-MAPPS samples as well as d-MAPPS-based eye drops, used in this study, were manufactured under specific conditions in order to be applicable for bioavailability testing and for different therapeutic use.

\section{Cells}

Therapeutic potential of d-MAPPS in corneal protection was determined by using human corneal epithelial cells (HCEC). HCEC was purchased from Gibco (catalog no. C018-5C). The cells were cultured in keratinocyte serum free medium (SFM) (17005-042) at $37^{\circ} \mathrm{C}$ in a $5 \% \mathrm{CO}_{2}$ incubator. $\mathrm{HCEC}$ in $2^{\text {nd }}$ passage was used throughout the experiment.

\section{In vitro induction of corneal epithelial cell injury}

HCEC were exposed to $1 \mathrm{~mL}$ of benzalkonium chloride (BAK) at concentrations of $0.001 \%$ and $0.005 \%$ for $30 \mathrm{~min}$. Control cells were treated with phosphate buffer saline [11]. HCEC were cultured for additional $48 \mathrm{~h}$ either in the presence of d-MAPPS or medium.

In order to evaluate whether d-MAPPS improved viability of BAK-injured HCEC, MTT (3-(4,5-dimethylthiazol-2yl)-2,5-diphenyltetrazolium bromide) test was used. 
HCEC were diluted with medium to $1 \times 10^{6}$ cells $/ \mathrm{ml}$ and were placed in individual wells in 96-multiplates. About $48 \mathrm{~h}$ later, after the cell adherence, each well received $100 \mu$ of $d-$ MAPPS or medium. Cells were incubated at $37^{\circ} \mathrm{C}$ in a $5 \%$ $\mathrm{CO}_{2}$ incubator for $24 \mathrm{~h}$. After incubation, multiplates were centrifuged, the supernatant was removed, fresh medium and MTT solution ( $5 \mathrm{mg} / \mathrm{ml}$ in PBS) $20 \mu$ were added to each well and the plates were incubated for an additional $4 \mathrm{~h}$. The multiplates were centrifuged, cell-free supernatants were suctioned off, and DMSO (150 $\mu$ l) and glycine buffer were added to dissolve the crystals. The plates were shaken for $10 \mathrm{~min}$. The optical density of each well was determined at $595 \mathrm{~nm}$ using microplate multimode detector Zenyth 3100. [12].

\section{Patients}

There was a total of 131 DED patients recruited ( 27 male and 104 female) with a median age of 62 years (range 1985). Patients received d-MAPPS eye drops and were followed-up for 12 months. The Principle of Good Clinical Practice and the Declaration of Helsinki were always adhered to. Patients were under continuous medical supervision by either their Ophthalmologist or Optometrist.

\section{Clinical assessment of d-MAPPS based effects}

Subjective symptoms were graded numerically using the VAS (visual analogue pain score). The scale ranged from 0 (absence of pain) to 10 (maximal pain). The subjects were asked to describe their discomfort or pain using the VAS. Standard Patient Evaluation of Eye Dryness Questionnaire (SPEED) is a questionnaire used for the evaluation of dry eye-related symptoms. The symptoms inquired by the SPEED questionnaire include dryness or grittiness or scratchiness, soreness or irritation, burning or watering, and eye fatigue reported and scored as sometimes-1, often-2, and constant-3, and whether these symptoms pose no problems0 , were tolerable-1, uncomfortable-2, bothersome-3, or intolerable-4 [13-14].

\section{Statistics}

Data were expressed as the mean \pm standard error of the mean (SEM) for each group. Results were analyzed by Student's t test. Statistical analyses were performed using SPSS 25.0 for Windows software (SPSS Inc., Chicago, IL, USA). The difference was considered significant when $\mathrm{p}<0.05$.

\section{RESULTS}

\section{d-MAPPS showed good tolerability on human corneal epithelial cell cultures}

In order to determine whether d-MAPPS was well tolerated by HCEC, these cells were cultured in the presence of this MSC-derived product. As it is shown in Figure 1A, dMAPPS-treatment was not toxic for HCEC. Morphology of HCEC was not altered by d-MAPPS and density of dMAPPS treated HCEC was not significantly lower when compared to the cells that were grown under standard culture conditions. Loss of cell-to-cell contact was noticed in BAKtreated HCEC, while d-MAPPS treated HCEC grew in the same manner as under standard culture conditions. Importantly, morphology and density of BAK-injured HCEC was remarkably improved after d-MAPPS treatment (Figure 1A).

Importantly, viability of BAK-injured HCEC that were cultured in the presence of d-MAPPS was significantly higher when compared to BAK-injured HCEC that were grown under standard culture conditions or AF. After 5 min exposure to BAK, the percentage of viable HCEC had decreased by approximately $25 \%$. After $15 \mathrm{~min}$ exposure to $\mathrm{BAK}$, the viability of HCEC decreased for additional $10 \%$, and at the $30 \mathrm{~min}$ time point, percentage of live cells significantly dropped down, being $48 \%$ lower than in the control, BAK-untreated group. Importantly, d-MAPPS treatment managed to significantly increase viability of BAK-injured HCEC at all time points (Figure 1B). The cell viability of BAK + d-MAPPS treated HCEC was $85 \%$ after 5 min exposure to $\mathrm{BAK}, 80 \%$ at the $10 \mathrm{~min}$ time point and percentage of viable HCEC was $65 \%$ after 30 min exposure to BAK (Figure 1B). d-MAPPS did not affect viability of BAK-untreated cells. There was no significant difference in the percentage of viable HCEC between BAK-untreated HCEC that grew under standard culture conditions and in the presence of dMAPPS (Figure 1B).

\section{d-MAPPS significantly attenuated VAS and SPEED scores in DED patients}

In line with the in vitro-observed results were findings obtained in clinical settings. Significantly reduced VAS (Figure 2A) and SPEED (Figure 2B) scores were noticed in dMAPPS-treated DED patients, indicating that d-MAPPS eye drops managed to improve symptoms including pain, dryness, grittiness, scratchiness, soreness, irritation, burning, watering and eye fatigue (Figure 2). Importantly, d-MAPPS induced beneficial effects have been noticed during the entire observational period and significantly increased during the last 6 months of the follow-up. Significantly lower VAS and SPEED scores were documented 3 months after d-MAPPS treatment (Figure $2 \mathrm{~A}-\mathrm{B}, \mathrm{p}<0.001$ ), but the highest reduction in VAS and SPEED scores in DED patients were observed after 12 months of d-MAPPS-based therapy, indicating the long-lasting beneficial effects of d-MAPPS in alleviation of ocular symptoms in DED patients. 
Figure 1. d-MAPPS showed good tolerability on human corneal epithelial cell cultures.
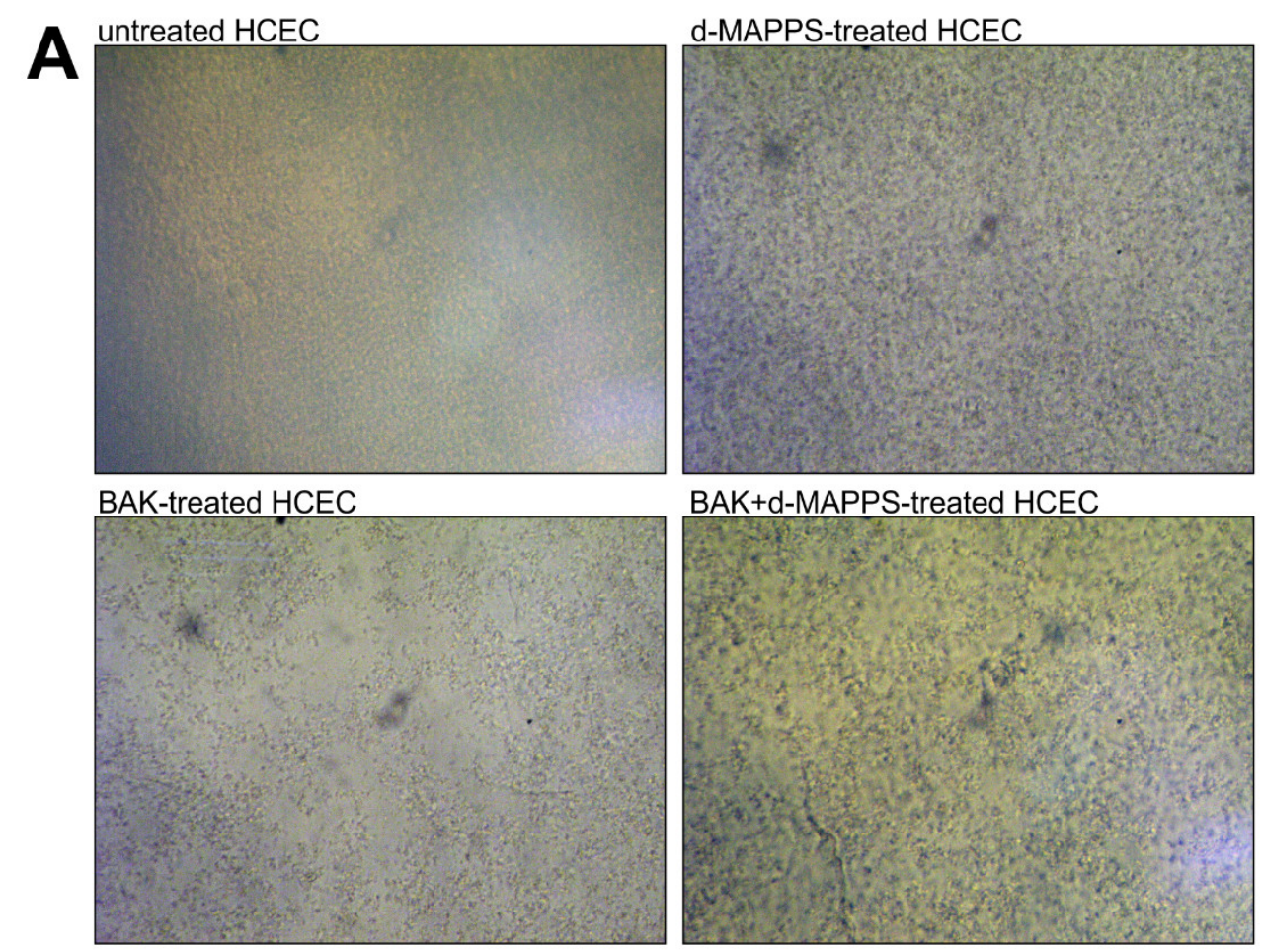

B

Human corneal epithelial cells

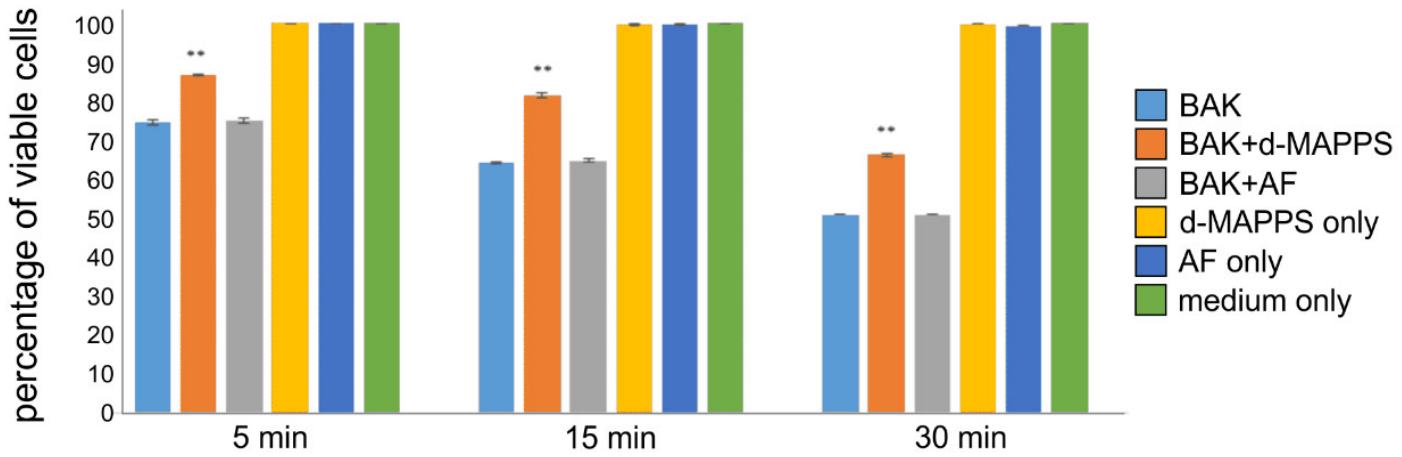

A) Morphology of human corneal epithelial cells (HCEC) was not altered by d-MAPPS. Density of d-MAPPS-treated HCEC was not significantly lower when compared to the cells that were grown under standard culture conditions. d-MAPPS significantly reduced benzalkonium chloride (BAK)-induced decrease in HCEC density.

B) Results obtained by MTT assay showed that viability of BAK + d-MAPPS treated HCEC was significantly higher when compared to BAK-injured HCEC that were grown under standard culture conditions or amniotic fluid (AF). Results are shown as Mean+/-standard deviation $(* * \mathrm{p}<0.01)$. 
Figure 2. d-MAPPS eye drops significantly improved clinical symptoms in DED patients.
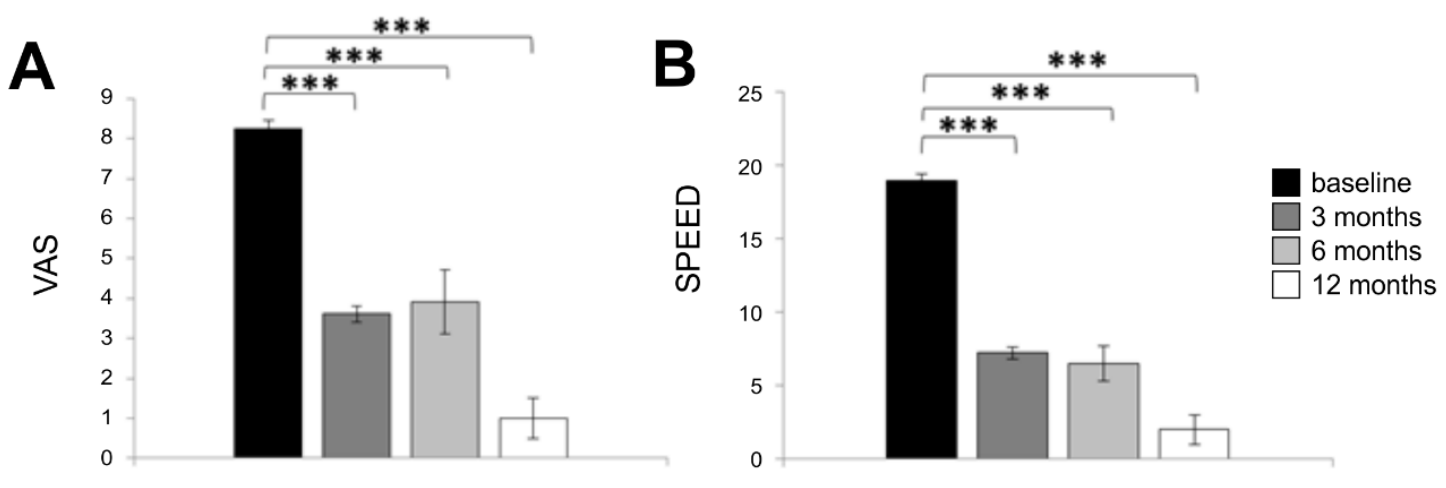

Significantly reduced (A) Visual Analogue pain (VAS) and

(B) Standard Patient Evaluation of Eye Dryness Questionnaire (SPEED) scores were noticed in 131 d-MAPPS treated DED patients, during the 12 month of follow-up $(* * * p<0.001)$.

\section{DISCUSSION}

Herewith, we demonstrated therapeutic potential of newly designed MSC-derived product d-MAPPS eye drops in attenuation of DED. d-MAPPS was well tolerated by corneal epithelial cells and promoted their recovery from BAKinduced injury (Figure 1). Accordingly, d-MAPPS eye drops efficiently alleviated ocular symptoms in DED patients, indicating therapeutic potential of d-MAPPS in suppression of inflammatory diseases of the eye.

Several lines of evidence indicated that $\mathrm{T}$ cell-driven immune response has crucial role in the pathogenesis of DED [15-17]. Furthermore, inflammation can be considered as a cause and a consequence of DED [18]. Alterations in tear production and composition, particularly elevated osmolarity, promote inflammation on the ocular surface and lid margins, by activating c-Jun N-terminal kinase (JNK) and NF-k $\beta$ signaling pathways in epithelial cells which result in enhanced secretion of alarmins, pro-inflammatory cytokines and chemokines $[15,18]$. Increased concentration of these mediators attracts circulating monocytes and lymphocytes into the lacrimal glands and ocular surface [18]. DCs and T cells within ocular surface and draining lymph nodes interact to defend the eye against the variety of microbial agents that populate or infect the cornea and ocular surface $[15,18]$. Resident DCs capture bacterial antigens, present them to the naive CD4+ T cells in regional lymph nodes and through the secretion of IL-12 induce their differentiation in effector, IFN- $\gamma$-producing Th1 cells which, in turn, in IFN- $\gamma$ dependent manner promote polarization of resident macrophages in inflammatory M1 phenotype [15]. Th1 cell-derived IFN- $\gamma$ promote apoptosis and squamous metaplasia of the ocular surface epithelia while M1 macrophage-derived matrix metalloproteinases (MMPs) and inflammatory mediators (tumor necrosis factor alpha (TNF- $\alpha$ ) and nitric oxide) disrupt epithelial cell barriers $[15,18]$. We recently demonstrated that d-MAPPS significantly attenuated concentration of IL12 in the supernatants of activated human peripheral blood mononuclear cells (pbMNCs) and alleviate production of IFN- $\gamma$ in activated lymphocytes [19]. d-MAPPS contains large number of immunoregulatory factors which are capable to suppress detrimental $\mathrm{T}$ cell-driven immune response [10]. Among them, GRO- $\gamma$ is mainly responsible for the suppression of DCs:T cell cross-talk and for inhibition of DCdependent generation of inflammatory Th1 cells [20]. Human MSCs secrete GRO- $\gamma$ which was found in high concentration in d-MAPPS samples [20]. MSCs, in GRO- $\gamma$-dependent manner induce polarization of monocyte-derived DCs into myeloid derived suppressor cells. GRO- $\gamma$-treated DCs had a tolerogenic phenotype characterized by increased secretion of immunosuppressive IL-10, and reduced production of inflammatory cytokines IL-12 and IFN- $\gamma$ [20]. In line with these findings, we assume that administration of GRO- $\gamma$ containing d-MAPPS eye drops, suppressed production of IL-12 in resident DCs, inhibited generation of Th1 lymphocytes and M1 macrophages and resulted in alleviation of eye inflammation in DED patients.

Inflammatory DCs in IL-1, IL-6 and IL-23-dependent manner induce differentiation of naïve $\mathrm{T}$ cells into effector Th17 cells that reduce tear production and promote progression of DED [18]. In similar manner as Th1 cells, effector Th17 cells promote corneal epithelial barrier disruption through the enhanced production of IL-17 [15]. Since dMAPPS efficiently attenuated production of IL-17 in activated CD4+ T helper cells [19], we assume that d-MAPPSinduced suppression of Th17 cell-driven inflammation in the eye was, at least partially, responsible for beneficial effects of d-MAPPS eye drops in DED patients. 
CD4+CD25+FoxP3+T regulatory cells (Tregs) have crucially important role in the suppression of eye inflammation in DED patients [21]. Reduced number of Tregs, usually accompanied with increased expansion of inflammatory CD4+ $\mathrm{T}$ cells (particularly Th17 cells) is observed in DED patients with aggravated diseases, while increased Tregs/Th17 cells is followed by tissue repair and regeneration [22-23]. Therefore, therapeutic agents that may induce generation and proliferation of Tregs have beneficial effects in attenuation of DED symptoms [23]. Among MSC-derived immunomodulatory factors, Indoleamine 2, 3-dioxygenase 1 (IDO1) was crucially responsible for the maintenance of immunosuppressive phenotype in resting Tregs [24-25]. During activation of resting Tregs, signals generated from activated $\mathrm{T}$ cell receptor, via mammalian target of rapamycin (mTOR) pathway, destabilize immunosuppressive phenotype of Tregs and cause their reprogramming into a pro-inflammatory helperlike phenotype ("ex-Tregs"), characterized by enhanced production of inflammatory cytokines, particularly IL-17 and IFN- $\gamma$ [25]. As emphasized by us and others, IDO1 maintains population of Tregs in inflamed tissue by preventing transdifferentiation of Tregs in inflammatory Th1 and Th17 cells [26-27]. IDO1 activates general control nonderepressible 2 (GCN2) kinase in activated Tregs which inhibits mTOR signaling and prevents destabilization of immunosuppressive phenotype of Tregs enabling their expansion [25]. Having in mind that elevated IDO1 activity was measured in d-MAPPS samples [10], we believe that IDO-1-dependent expansion of Tregs could be, at least partially, responsible for beneficial effects of d-MAPPS eye drops in DED patients.

\section{CONCLUSION}

Due to their immunosuppressive and regenerative properties, d-MAPPS eye drops represent potentially new therapeutic agents that may efficiently alleviate eye inflammation and improve quality of life of DED patients.

\section{ETHICS APPROVAL AND CONSENT TO PARTICIPATE}

The study was conducted in accordance with the ethical standards of the committee responsible for human experimentation (institutional and national) and the Helsinki Declaration of 1975, as revised in 2013. Voluntary written and informed consent was obtained from each participant prior to enrollment in the study.

\section{COMPETING INTERESTS}

There are no conflicts of interest.

\section{FUNDING}

This study was supported by Faculty of Medical Sciences University of Kragujevac, Serbia (Grant MP01/18).

\section{LITERATURE}

1. Craig JP, Nichols KK, Akpek EK, Caffery B, Dua HS, Joo CK, Liu Z, Nelson JD, Nichols JJ, Tsubota K, Stapleton F. TFOS DEWS II Definition and Classification Report. Ocul Surf. 2017;15:276-283.

2. Milner MS, Beckman KA, Luchs JI, Allen QB, Awdeh RM, Berdahl J, Boland TS, Buznego C, Gira JP, Goldberg DF, Goldman D, Goyal RK, Jackson MA, Katz J, Kim T, Majmudar PA, Malhotra RP, McDonald MB, Rajpal RK, Raviv T, Rowen S, Shamie N, Solomon JD, Stonecipher K, Tauber S, Trattler W, Walter KA, Waring GO 4th, Weinstock RJ, Wiley WF, Yeu E. Dysfunctional tear syndrome: dry eye disease and associated tear film disorders - new strategies for diagnosis and treatment. Curr Opin Ophthalmol. 2017;27 Suppl 1:3-47.

3. Foulks GN, Forstot SL, Donshik PC, Forstot JZ, Goldstein MH, Lemp MA, Nelson JD, Nichols KK, Pflugfelder SC, Tanzer JM, Asbell P, Hammitt K, Jacobs DS. Clinical guidelines for management of dry eye associated with Sjögren disease. Ocul Surf. 2015;13:118-32.

4. Stevenson W, Chauhan SK, Dana R. Dry eye disease: an immune-mediated ocular surface disorder. Arch Ophthalmol. 2012;130:90-100.

5. Messmer EM. The pathophysiology, diagnosis, and treatment of dry eye disease. Dtsch Arztebl Int. 2015;112:71-81.

6. Nguyen LS, Vautier M, Allenbach Y, Zahr N, Benveniste O, Funck-Brentano C, Salem JE. Sirolimus and mTOR Inhibitors: A Review of Side Effects and Specific Management in Solid Organ Transplantation. Drug Saf. 2019;42:813-825.

7. Marshall LL, Roach JM. Treatment of Dry Eye Disease. Consult Pharm. 2016;31:96-106.

8. Villatoro AJ, Fernández V, Claros $\mathrm{S}$, Alcoholado $\mathrm{C}$, Cifuentes M, Merayo-Lloves J, Andrades JA, Becerra J. Regenerative Therapies in Dry Eye Disease: From Growth Factors to Cell Therapy. Int $\mathrm{J}$ Mol Sci. 2017;18(11). pii: E2264.

9. Harrell CR, Simovic Markovic B, Fellabaum C, Arsenijevic A, Djonov V, Arsenijevic N, Volarevic V. Therapeutic Potential of Mesenchymal Stem Cell-Derived Exosomes in the Treatment of Eye Diseases. Adv Exp Med Biol. 2018;1089:47-57.

10. Harrell CR, Fellabaum C, Simovic Markovic B, Arsenijevic A, Volarevic V. Therapeutic potential of "Exosomes derived Multiple Allogeneic Proteins Paracrine Signaling: Exosomes d-MAPPS" is based on the effects of exosomes, immunosuppressive and trophic factors. Ser J of Exp Clin Res. 2018; doi:10.2478/sjecr-20180032 .

11. Cha SH, Lee JS, Oum BS, Kim CD. Corneal epithelial cellular dysfunction from benzalkonium chloride (BAC) in vitro. Clin Exp Ophthalmol. 2004; 32:180-184.

12. Arsenijevic M, Milovanovic M, Jovanovic S, Arsenijevic N, Markovic BS, Gazdic M, Volarevic V. In vitro and in vivo anti-tumor effects of selected platinum(IV) and dinuclear platinum(II) complexes against lung cancer cells. J Biol Inorg Chem. 2017;22:807-817. 
13. Asiedu K, Kyei S, Mensah SN, Ocansey S, Abu LS, Kyere EA. Ocular surface disease index (OSDI) versus the standard patient evaluation of eye dryness (SPEED): a study of a nonclinical sample. Cornea 2016; 35:175180.

14. Finis D, Pischel N, König C, Hayajneh J, Borrelli M, Schrader S, Geerling G. Comparison of the OSDI and SPEED questionnaires for the evaluation of dry eye disease in clinical routine. Ophthalmologe. 2014;111:10501056.

15. Pflugfelder SC, Corrales RM, de Paiva CS. T helper cytokines in dry eye disease. Exp Eye Res. 2013;117: 118-25.

16. Semba CP, Gadek TR. Development of lifitegrast: a novel T-cell inhibitor for the treatment of dry eye disease. Clin Ophthalmol. 2016;10:1083-94.

17. Dohlman TH, Ding J, Dana R, Chauhan SK. T Cell-Derived Granulocyte-Macrophage Colony-Stimulating Factor Contributes to Dry Eye Disease Pathogenesis by Promoting CD11b+ Myeloid Cell Maturation and Migration. Invest Ophthalmol Vis Sci. 2017;58:1330-1336.

18. Wei Y, Asbell PA. The core mechanism of dry eye disease is inflammation. Eye Contact Lens. 2014;40:248-56.

19. Harrell CR, Simovic Markovic B, Fellabaum C, Miloradovic D, Acovic A, Miloradovic D, Arsenijevic N, Volarevic V. Exo-d-MAPPS attenuates production of inflammatory cytokines and promotes generation of immunosuppressive phenotype in peripheral blood mononuclear cells. Ser J of Exp Clin Res. 2019; doi: 10.2478/sjecr-2019-0045.

20. Chen HW, Chen HY, Wang LT, Wang FH, Fang LW, Lai HY, Chen HH, Lu J, Hung MS, Cheng Y, Chen MY, Liu SJ, Chong P, Lee OK, Hsu SC. Mesenchymal stem cells tune the development of monocyte-derived dendritic cells toward a myeloid-derived suppressive phenotype through growth-regulated oncogene chemokines. J Immunol. 2013;190:5065-77.

21. Ratay ML, Glowacki AJ, Balmert SC, Acharya AP, Polat J, Andrews LP, Fedorchak MV, Schuman JS, Vignali DAA, Little SR. Treg-recruiting microspheres prevent inflammation in a murine model of dry eye disease. $\mathrm{J}$ Control Release. 2017;258:208-217.

22. Chauhan SK, El Annan J, Ecoiffier T, Goyal S, Zhang Q, Saban DR, Dana R. Autoimmunity in dry eye is due to resistance of Th17 to Treg suppression. J Immunol. 2009; 182:1247-52.

23. Fu R, Jiang Y, Zhou J, Zhang J. Rebamipide ophthalmic solution modulates the ratio of $T$ helper cell 17/regulatory $\mathrm{T}$ cells in dry eye disease mice. Mol Med Rep. 2019; 19:4011-4018.

24. Ge W, Jiang J, Arp J, Liu W, Garcia B, Wang H. Regulatory T-cell generation and kidney allograft tolerance induced by mesenchymal stem cells associated with indoleamine 2,3-dioxygenase expression. Transplantation. 2010;90:1312-20.

25. Harrell CR, Jankovic MG, Fellabaum C, Volarevic A, Djonov V, Arsenijevic A, Volarevic V. Molecular Mechanisms Responsible for Anti-inflammatory and
Immunosuppressive Effects of Mesenchymal Stem CellDerived Factors. Adv Exp Med Biol. 2019;1084:187206.

26. Volarevic V, Zdravkovic N, Harrell CR, Arsenijevic N, Fellabaum C, Djonov V, Lukic ML, Simovic Markovic B. Galectin-3 Regulates Indoleamine-2,3-dioxygenaseDependent Cross-Talk between Colon-Infiltrating Dendritic Cells and T Regulatory Cells and May Represent a Valuable Biomarker for Monitoring the Progression of Ulcerative Colitis. Cells. 2019 Jul 12;8(7). pii: E709.

27. Matteoli G, Mazzini E, Iliev ID, Mileti E, Fallarino F, Puccetti P, Chieppa M, Rescigno M. Gut CD103+ dendritic cells express indoleamine 2,3-dioxygenase which influences $\mathrm{T}$ regulatory $/ \mathrm{T}$ effector cell balance and oral tolerance induction. Gut. 2010;59(5):595-604. 